\title{
UsiWSC: Framework for Supporting an Interactive Web Service Composition ${ }^{\star}$
}

\author{
Mohamed Boukhebouze, Waldemar P. Ferreira Neto, \\ Erbin Lim, and Philippe Thiran \\ PReCISE Research Center, University of Namur, 5000, Belgium \\ \{mboukheb, waldemar.neto, erbin.lim, philippe.thiran\}@fundp.ac. be
}

\begin{abstract}
In this paper, we propose the UsiWSC framework for designing, deploying and executing Web service compositions with user interactions. The UsiWSC design relies on an extension of the BPEL standard: UI-BPEL. The extension supports the derivation of user interfaces as well as executable compositions for different user contexts. The UsiWSC execution requires the coordination of the control and data flows between web services and user interfaces.
\end{abstract}

Keywords: Interactive Web Service Composition, BPEL, User Interface, User Interaction.

\section{Introduction}

A Web service composition is the process that orchestrates or choreographs a set of Web services in order to implement a business process [8]. This mechanism is used to fulfill the user request that a single Web service cannot satisfy 8]. Several initiatives have been adopted to provide languages that allow the description of Web service composition execution. Nowadays, WS-BPEL (BPEL for short) [7] has become the de facto standard in the industry [8]. This standard is intended to express a composition process in a fully automated way. As such, users are not able to interact with the Web services until the end of the process execution: they cannot provide input to a Web service at runtime, they cannot cancel the process execution, nor they can see some intermediary output from a Web service. However, many Web service composition scenarios require user interactions [6].

In this work, we propose the UsiWSC framework (Usable uSer Interface for Web Service Composition) that supports the design, the deployment and the execution of an interactive Web service composition. At design time, an interactive composition is defined based on an UI-BPEL extension [2] that supports the specification of user roles and the different types of user interactions. At deployment time, abstract user interfaces and BPEL are derived from UI-BPEL. Abstract user interfaces (AUI) and BPEL are not intended to be executed: they

\footnotetext{
^ Research supported by la Wallonie.
} 
are independent of any user context and can therefore be adapted for different use cases. At runtime, user contexts are used for providing concrete user interfaces (CUI) and executable BPEL.

UsiWSC is part of several initiatives to enhance BPEL with user interactions. An example of such an initiative is ActiveBPEL for People [1. The ActiveBPEL for People framework describes an interactive WSC based on an extension of BPEL called BPEL4People 6]. BPEL4People introduces a new type of BPEL activity to specify user tasks. ActiveBPEL for People framework also supports the generation of user interfaces according to user task parameters. Another initiative is BPEL4UI [3]. This framework relies on an extension of BPEL in which Partner Links can be used to connect BPEL activities and user interfaces. User interfaces are developed separately from the composition. UsiWSC differs from these main initiatives in that (1) UI-BPEL of UsiWSC supports the user event interaction (a process can be cancelled by the user at any time of the composition); and (2) UsiWSC enables the generation of user interfaces adapted to the user contexts.

The rest of the paper is organized as follows. Section 2 describes the UsiWSC architecture by focusing on its main software components. Section 3 presents the online demonstration scenario that is based on a simple purchase order process.

\section{UsiWSC Architecture}

The UsiWSC architecture is presented in Figure 1] The figure shows the major software components (namely, the UI-BPEL designer, the transformation tools and the UI manager) and their interactions. Software components are classified according to two different views: the abstraction level (abstract or concrete) and the modeling object (user interface or Web service composition).

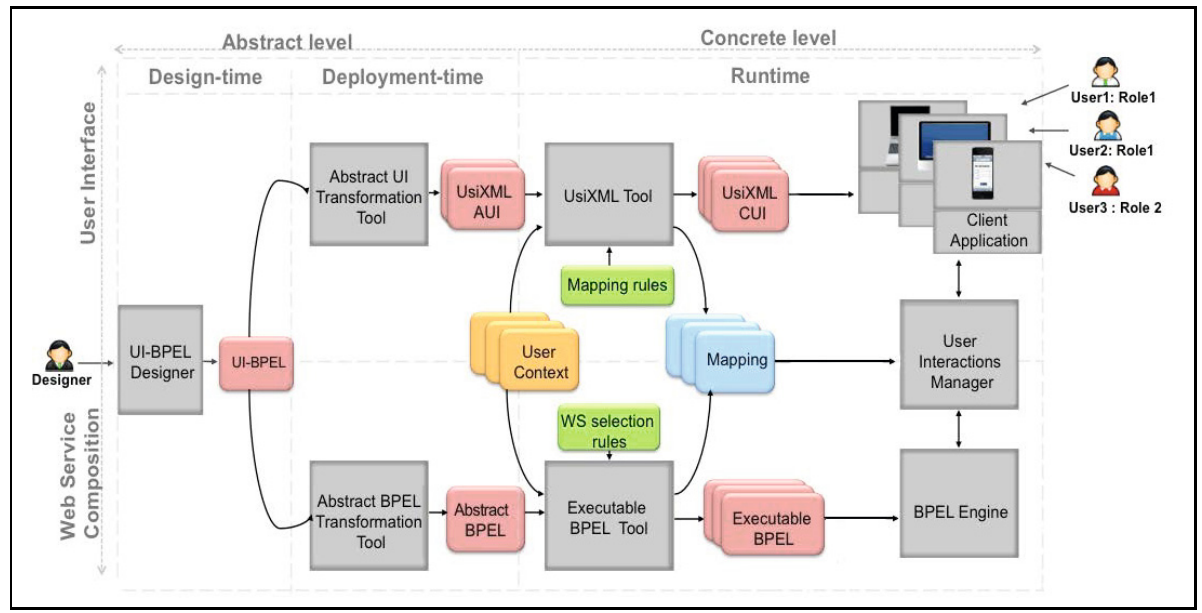

Fig. 1. Overview of the UsiWSC Architecture 


\subsection{UI-BPEL Designer}

The UI-BPEL Designer is a tool that supports the design of a UI-BPEL process. This tool is developed as an Eclipse plug-in based on the Eclipse BPEL Designer 4. The UI-BPEL specification enables the description of different user interactions by introducing new BPEL elements 2: (1) a new set of BPEL activities (DataInputUI, DataOutputUI, DataSelectionUI) to express different user data interactions; (2) a new type of BPEL events (InteractionEventUI) to express the interaction event; (3) an extension of the BPEL's Pick and Scope activities that supports the new InteractionEventUI; (4) a new activity attribute UserRole that specifies the role that is assigned to a data interaction activity.

\subsection{Transformation Tools}

From an UI-BPEL process, the UsiWSC framework derives both a valid BPEL and the related user interfaces, first at an abstract level, and then at a concrete level. The user interfaces are described as AUI and CUI by using the UsiXML language [5] while the abstract and executable BPEL are compliant with the BPEL specification 77. UsiXML has been chosen due to its good expressiveness to describe the different facets of user interfaces. In addition, UsiXML is in the process of being standardized by W3C.

The abstract UI transformation tool generates an AUI for each UI-BPEL role directly from an UI-BPEL process. The generated AUI describes UI independently to any user context (user preference, user device or user environment). As such, the transformation rules apply for each role and each UI-BPEL user interactions for converting them into a set of abstract UsiXML compounds (e.g. output abstract compounds, and output abstract compounds). CUI are obtained from the AUI by applying a UsiXML transformation tool. For each abstract compound, the UsiXML transformation tool creates a concrete compound based on the user context. For instance, an output abstract compound can be transformed into a label, which is expressed in the user preference language. The UsiXML transformation tool also keeps track of the transformations so that mappings can be defined between the executable BPEL and the different CUI as explained below.

The abstract BPEL transformation tool derives an abstract BPEL from the UI-BPEL. The generated abstract BPEL describes the control flow of the composition independently to any concrete Web service. Moreover, the abstract BPEL transforms each user interaction to an invocation of a particular Web service, namely the UI Manager (Section 2.3). An executable BPEL can then be generated by selecting component Web services that are involved in the composition. This selection is based on the user context and a set of predefined Web service selection rules. Note that, the Web service selection is not in the scope of this demonstration. 


\subsection{UI Manager}

The UI manager routes data between the executable BPEL and its CUI. The routing is derived from the mappings that are automatically generated from the UsiXML transformation tool. When an executable BPEL process invokes the UI manager, the UI manager relies on the mappings between the related UI-BPEL interactions and the CUI compounds for specifying the related CUI. This CUI is displayed as a final user interface to a client application. When the user interaction is completed (e.g., data input are provided by the user), the UI manager sends the user interaction result (user data or event) to the BPEL engine.

\section{Demonstration}

The scenario used in the demonstration is a simple purchase order process which involves different users with different roles and contexts of use. Though simple, the demonstration scenario outlines the main features of the UsiWSC framework: (1) user interactions modeling with the UI-BPEL editor (e.g. the shipper is manually selected by an administrator (data selection)); (2) multi-role and multi-context user interfaces generation as well as an executable BPEL process generation; and (3) runtime coordination between the WSC and its UI in multicontext (different user devices). The demonstration video is available at: http://webapps.fundp.ac.be/usiwsc/index.html \& http://www.youtube.com/watch?v=_py7E9zqqg4

\section{References}

1. Active-Endpoint: ActiveBPEL 4 People (2007), http://www.activebpel.org/ samples/samples-4/ActiveBPELforPeople/doc/index.html

2. Boukhebouze, M., Neto, W.P.F., Erbin, L.: Yet Another BPEL Extension for User Interactions. In: De Troyer, O., Bauzer Medeiros, C., Billen, R., Hallot, P., Simitsis, A., Van Mingroot, H. (eds.) ER Workshops 2011. LNCS, vol. 6999, pp. 24-33. Springer, Heidelberg (2011)

3. Daniel, F., Soi, S., Tranquillini, S., Casati, F., Heng, C., Yan, L.: From People to Services to UI: Distributed Orchestration of User Interfaces. In: Hull, R., Mendling, J., Tai, S. (eds.) BPM 2010. LNCS, vol. 6336, pp. 310-326. Springer, Heidelberg (2010)

4. Eclipse-Fundation: Eclipse bpel designer (2011), http://www.eclipse.org/bpel/

5. ITEA2: UsiXML Project (2009), http://www .usixml.eu

6. Kloppmann, M., Koenig, D., Leymann, F., Pfau, G., Rickayzen, A., von Riegen, C., Schmidt, P., Trickovic, I.: Ws-bpel extension for people-bpel4people. Joint White Paper, IBM and SAP (2005)

7. OASI: Web Services Business Process Execution Language(BPEL) 2.0. wsbpelspecification-draft-01 (2007)

8. Zeng, L., Benatallah, B., Ngu, A.H.H., Dumas, M., Kalagnanam, J., Chang, H.: Qos-aware middleware for web services composition. IEEE Trans. Softw. Eng. 30, 311-327 (2004) 